\title{
TITLE:
}

\section{Partial restoration of chiral symmetry in the color flux tube}

$\operatorname{AUTHOR}(\mathrm{S})$ :

Iritani, Takumi; Cossu, Guido; Hashimoto, Shoji

CITATION:

Iritani, Takumi ...[et al]. Partial restoration of chiral symmetry in the color flux tube. Physical Review D 2015, 91(9): 094501.

ISSUE DATE:

2015-05-05

URL:

http://hdl.handle.net/2433/201407

RIGHT:

C 2015 American Physical Society 
PHYSICAL REVIEW D 91, 094501 (2015)

\title{
Partial restoration of chiral symmetry in the color flux tube
}

\author{
Takumi Iritani, ${ }^{1, *}$ Guido Cossu, ${ }^{2}$ and Shoji Hashimoto ${ }^{2,3}$ \\ ${ }^{1}$ Yukawa Institute for Theoretical Physics, Kyoto University, Kitashirakawa-oiwake, Sakyo, \\ Kyoto 606-8502, Japan \\ ${ }^{2}$ High Energy Accelerator Research Organization (KEK), Tsukuba, Ibaraki 305-0801, Japan \\ ${ }^{3}$ School of High Energy Accelerator Science, The Graduate University for Advanced Studies (Sokendai), \\ Tsukuba, Ibaraki 305-0801, Japan
}

(Received 18 February 2015; published 5 May 2015)

\begin{abstract}
Using the quark eigenmodes computed on the lattice with the overlap-Dirac operator, we investigate the spatial distribution of the chiral condensate around static color sources corresponding to quark-antiquark and three-quark systems. A flux structure of chromo fields appears in the presence of such color charges. The magnitude of the chiral condensate is reduced inside the color flux, which implies partial restoration of chiral symmetry inside hadrons. Taking a static baryon source in a periodic box as a toy model of nuclear matter, we estimate the magnitude of the chiral symmetry restoration as a function of baryon matter density.

DOI: 10.1103/PhysRevD.91.094501

PACS numbers: 12.38.Gc, 11.30.Rd, 12.38.Aw
\end{abstract}

\section{INTRODUCTION}

The low-energy dynamics of QCD is characterized by two prominent properties, i.e., chiral symmetry breaking and confinement. In the QCD vacuum, chiral symmetry of quark fields is spontaneously broken, as probed by an order parameter $\langle\bar{q} q\rangle$, the vacuum expectation value of the scalar density operator $\bar{q} q$. Through the Banks-Casher relation [1], this chiral condensate $\Sigma=-\langle\bar{q} q\rangle$ can be related to the spectral density of the low-lying Dirac eigenvalues. In the presence of valence quarks, or color sources, some modification of the vacuum is expected around them. Strictly speaking, it is no longer the vacuum, i.e., the lowest energy state of the system, but we use this terminology having in mind an application to the study of finite density QCD. Although the static color sources as considered in this work are only a crude approximation to the finite density system, the study of the vacuum modification may shed light on the states of finite-density QCD, which has been a subject of active research (see, for instance, Ref. [2]).

The other interesting property of low-energy QCD is the confinement of quarks, which is characterized by the linearly rising potential between static color sources. Putting a pair of a static quark and antiquark in the vacuum, a color flux tube emerges between them and leads to a linear increase of the energy as a function of the separation. This flux-tube structure has been observed in lattice QCD calculations by monitoring the action density or chromoelectric (or chromomagnetic) field [3-5]. We expect that such a flux-tube structure is reflected in the low-lying fermion eigenmodes because the Dirac eigenmodes carry the information of their background gauge field configuration. Indeed, the QCD field-strength tensor can be reconstructed using the fermion eigenmodes [6].

*iritani@yukawa.kyoto-u.ac.jp
In this paper, we present a lattice study of the spatial distribution of the chiral condensate in the presence of static color charges. We consider quark-antiquark and three-quark systems represented by Wilson loops to mimic the mesonic and baryonic states, respectively. We use the lattice data of the Dirac eigenmodes calculated on the gauge configurations generated with $2+1$-flavor dynamical overlap fermions [7]. With the overlap fermion formulation $[8,9]$, chiral symmetry is exactly realized on the lattice, which is important in the study of the low-lying Dirac eigenmodes, as they are very sensitive to any small violations of chiral symmetry. The lattice data used in this work have this nice property and indeed were successfully applied to the extraction of the chiral condensate in the vacuum [10-13].

The organization of this paper is as follows. In Sec. II, we describe the method to construct the local chiral condensate $\bar{q} q(x)$ by using the overlap-Dirac eigenmodes and show its distribution in the vacuum. In Secs. III and IV, we investigate the spatial distribution of the local chiral condensate around the static color sources. Section V is devoted to a summary. Preliminary reports of this work are found in Refs. [14-16].

\section{TOPOLOGICAL STRUCTURE OF THE QCD VACUUM}

We investigate the topological structure of the nonperturbative QCD vacuum in terms of the eigenmodes of the overlap-Dirac operator. It preserves exact chiral symmetry, and the relation to the topological charge of background gauge field configuration is manifest, i.e., the index theorem, at least for smooth enough backgrounds [17].

In this paper, we use the $2+1$-flavor dynamical overlapfermion configurations generated by the JLQCD Collaboration [7]. Their lattice volumes are $16^{3} \times 48$ and 
$24^{3} \times 48$ at a single inverse lattice spacing $a^{-1}=$ $1.759(10) \mathrm{GeV}$. The dynamical quark masses are $m_{u d}=$ $0.015 a^{-1}$ and $m_{s}=0.080 a^{-1}$. The global topological charge is fixed at $Q=0$ to avoid the problem of divergent molecular dynamics force in the simulations [18]. It induces finite-volume effects [19], which would not be significant for the relatively local observables considered in this study. Most of the results are obtained on the larger lattice $\left(24^{3} \times 48\right)$ where the number of independent configurations is 50 .

In the following, we describe the profile of the low-lying eigenmodes on these lattices.

\section{A. Local chiral condensate $\bar{q} q(x)$}

The massless overlap-Dirac operator is given by $[8,9]$

$$
D_{\text {ov }}(0)=m_{0}\left[1+\gamma_{5} \operatorname{sgn} H_{W}\left(-m_{0}\right)\right]
$$

with the Hermitian Wilson-Dirac operator $H_{W}\left(-m_{0}\right)=$ $\gamma_{5} D_{W}\left(-m_{0}\right)$. Here, sgn denotes the matrix sign function. Introducing the quark mass $m_{q}$, the overlap-Dirac operator is modified as

$$
D_{\mathrm{ov}}\left(m_{q}\right)=\left(1-\frac{m_{q}}{2 m_{0}}\right) D_{\mathrm{ov}}(0)+m_{q}
$$

This form cancels $\mathcal{O}(a)$ discretization effects, together with a proper rotation of the fermion fields in the observables.

We define the eigenfunction $\psi_{\lambda}(x)$ associated with an eigenvalue $\lambda$ of the massless overlap-Dirac operator

$$
D_{\mathrm{ov}}(0) \psi_{\lambda}(x)=\lambda \psi_{\lambda}(x),
$$

where the eigenfunction $\psi_{\lambda}(x)$ is normalized as $\sum_{x} \psi_{\lambda}^{\dagger}(x) \psi_{\lambda}(x)=1$. Using the eigenfunctions, we may expand the "local chiral condensate" $\bar{q} q(x)$ in terms of the eigenmodes, i.e.,

$$
\bar{q} q(x)=-\sum_{\lambda} \frac{\psi_{\lambda}^{\dagger}(x) \psi_{\lambda}(x)}{m_{q}+\left(1-\frac{m_{q}}{2 m_{0}}\right) \lambda}
$$

for a valence quark mass $m_{q}$. This relation represents a self-contracting fermion loop contribution from and to the scalar density operator. If the measured observables do not include other light quark fields to be contracted, then the substitution (4) is justified. The correlation functions of $\bar{q} q(x)$ with the Wilson loop are in this class of observables.

The chiral condensate $\langle\bar{q} q\rangle$ is given by an ensemble average of $\bar{q} q(x)$ without insertions of other operators. By averaging over space-time, this quantity is simply written in terms of only the eigenvalues because of the normalization condition for $\psi_{\lambda}(x)$. Thus, the relation between the chiral condensate and the spectral density $\rho(\lambda)$ of the Dirac eigenvalues is established. In the chiral limit, it reads $\Sigma=\pi \rho(0)$, i.e., the Banks-Casher relation [1].

\section{B. Action and topological charge densities in terms of the Dirac eigenmode}

Since the gauge field-strength tensor $F_{\mu \nu}$ is defined through the covariant derivative $D_{\mu}$ as $F_{\mu \nu}=\left[D_{\mu}, D_{\nu}\right]$, it can also be related to the Dirac operator [6]. Here, we briefly reproduce the derivation.

The square of the Dirac operator $\not D \equiv \gamma_{\mu} D_{\mu}$ is decomposed as

$$
[\not D(x)]^{2}=\sum_{\mu} D_{\mu}^{2}(x)+\sum_{\mu<\nu} \gamma_{\mu} \gamma_{\nu} F_{\mu \nu}(x) .
$$

By multiplying $\gamma_{\mu} \gamma_{\nu}$ and taking a trace with respect to the Dirac indices, the field-strength tensor is expressed as

$$
F_{\mu \nu}(x)=-\frac{1}{4} \operatorname{tr}\left[\gamma_{\mu} \gamma_{\nu} D^{2}(x)\right] .
$$

Therefore, by expanding the Dirac operator in terms of its eigenvectors $\psi_{\lambda}(x)$, an expansion of the field strength is obtained:

$$
F_{\mu \nu}(x)=\sum_{\lambda} \lambda^{2} f_{\mu \nu}(x)_{\lambda}, \quad f_{\mu \nu}(x)_{\lambda} \equiv \frac{i}{2} \psi_{\lambda}^{\dagger}(x) \gamma_{\mu} \gamma_{\nu} \psi_{\lambda}(x) .
$$

Using this decomposition, the action and topological charge densities are expressed as

$$
\rho(x)=\operatorname{tr}_{\mathrm{c}}\left[F_{\mu \nu} F_{\mu \nu}\right]=\operatorname{tr}_{\mathrm{c}} \sum_{\lambda, \lambda^{\prime}} \lambda^{2} \lambda^{\prime 2} f_{\mu \nu}(x)_{\lambda} f_{\mu \nu}(x)_{\lambda^{\prime}},
$$

$q_{\text {top }}(x)=\operatorname{tr}_{\mathrm{c}}\left[F_{\mu \nu} \tilde{F}_{\mu \nu}\right]=\operatorname{tr}_{\mathrm{c}} \sum_{\lambda, \lambda^{\prime}} \lambda^{2} \lambda^{\prime 2} f_{\mu \nu}(x)_{\lambda} \tilde{f}_{\mu \nu}(x)_{\lambda^{\prime}}$,

respectively. Here, $\operatorname{tr}_{c}$ denotes the trace with respect to the color indices, and $\tilde{f}_{\mu \nu}(x)_{\lambda}=\frac{1}{2} \varepsilon_{\mu \nu \rho \sigma} f_{\rho \sigma}(x)_{\lambda}$.

So far, the expressions are exact, but in the numerical studies, we introduce a truncation of the summation over the eigenmodes. This truncation acts as a filter to cut UV fluctuations above $\lambda_{\max }$. On the ensembles of $16^{3} \times 48$ and $24^{3} \times 48$ lattices, we calculated 160 and 240 pairs of eigenvalues and eigenvectors of $D_{\text {ov }}$, respectively. Then, the eigenvalues after correcting the $O(a)$ effect, $\operatorname{Im} \lambda /\left(1-\operatorname{Re} \lambda / 2 m_{0}\right)$, cover the region between $\pm 300 \mathrm{MeV}$, as shown in Fig. 1. In the measurements of the correlation between $\bar{q} q(x)$ and the Wilson loops, we monitor the dependence on the number $N$ of the eigenmodes included and confirm that the results saturate at least above $200 \mathrm{MeV}$. Some examples will be shown later.

Before presenting the results, we show some snapshots of the eigenmodes. The index theorem dictates that exact 


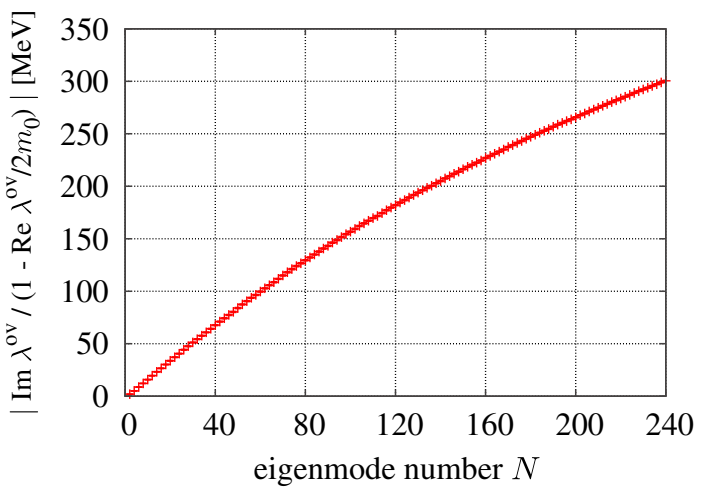

FIG. 1 (color online). Eigenvalue (in $\mathrm{MeV}$ ) of the low-lying eigenmodes on the $24^{3} \times 48$ lattice. By including 240 eigenmodes, we can cover the range of $\lambda \lesssim 300 \mathrm{MeV}$.

zero modes are associated with topological excitations of the gauge field. This suggests that the near-zero modes are superpositions of such local topological objects. Using a truncation at $N=20$, we visualize the low-mode contributions to the local chiral condensate $\bar{q} q(x)$ (4), the action density $\rho(x)(8)$, and the topological charge density $q_{\text {top }}(x)$ (9) in the panels (a), (b), and (c) of Fig. 2, respectively. They show tomographic images on a certain $T$ - $X$ slice of the four-dimensional lattice extracted from a given gauge configuration of size $24^{3} \times 48$.

As one can see in Fig. 2(a), the local condensate $\bar{q} q(x)$ forms a cluster structure. At the same space-time points of the cluster, the action density shows peaks [panel (b)]. More importantly, the topological charge density has positive and

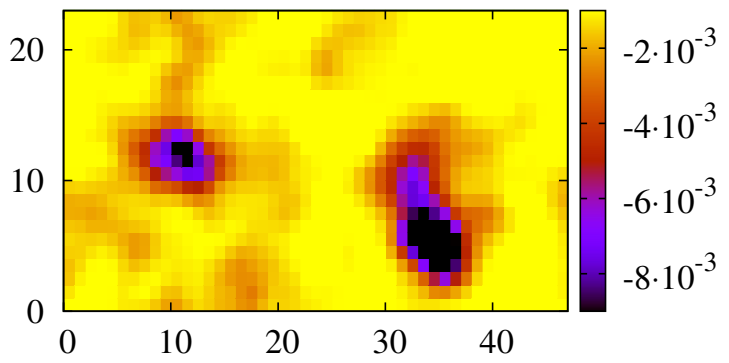

(a) negative islands stretching over several lattice spacings at the same space-time points. Such observation is not new; indeed, there are lattice studies using the overlap-Dirac operator $[20,21]$ showing the similar profile of the low-lying eigenmodes.

\section{CHIRAL CONDENSATE IN QUARK-ANTIQUARK SYSTEM}

In the presence of color charges, there appears a flux tube of chromoelectric fields, which has been observed on the lattice by measuring the spatial distribution of the field strength tensor [3-5]. In this section, we investigate the spatial distribution of the local chiral condensate $\bar{q} q(x)$ (4) around the static color sources. Previously, a related analysis has been made, but on a single color source, i.e., a Polyakov line [22-24], or at finite temperature at which the flux tube is expected to be suppressed [25].

\section{A. Partial restoration of the chiral symmetry in the flux tube}

We investigate the spatial distribution of the local chiral condensate $\bar{q} q(x)$ around the static color sources by calculating a correlation,

$$
\langle\bar{q} q(\vec{x})\rangle_{W} \equiv \frac{\langle\bar{q} q(\vec{x}) W(R, T)\rangle}{\langle W(R, T)\rangle}-\langle\bar{q} q\rangle
$$

where $W(R, T)$ denotes a Wilson loop of size $R \times T$. It represents a pair of a static quark and antiquark separated

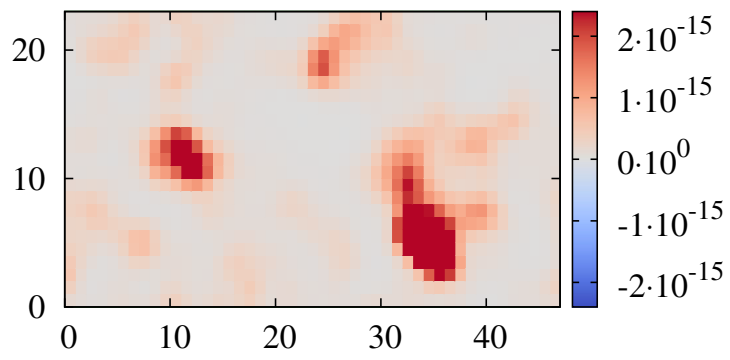

(b)

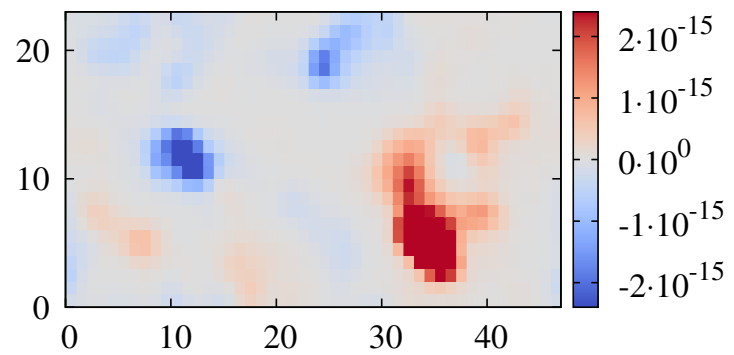

(c)

FIG. 2 (color online). Snapshots of (a) the local chiral condensate, (b) the action density, and (c) the topological charge distributions observed with the sum of 20 lowest-lying eigenmodes. These pictures show the same $T$ - $X$ slice of a $24^{3} \times 48$ lattice on a representative gauge configuration. The chiral condensate local fluctuations are correlated with the local topological charge measurements. 


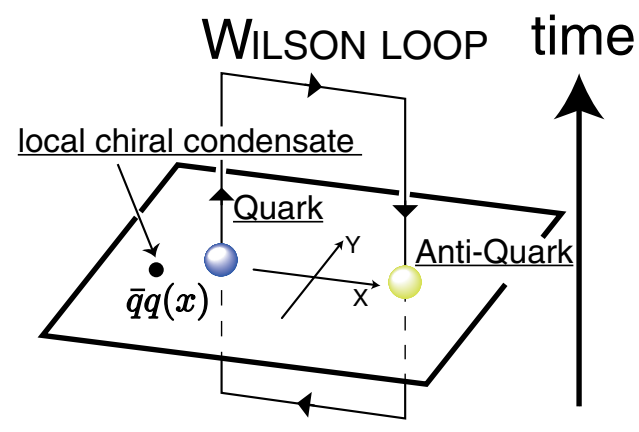

FIG. 3 (color online). Schematic picture of the flux-tube measurement. The static quark and antiquark are located at $(R / 2,0)$ and $(-R / 2,0)$ on the $X Y$ plane.

by a distance $R$. The origin of the coordinate is chosen at the center of the loop, which stretches along the $X$ axis, and the $Y$ and $Z$ axes correspond to the transverse directions. Figure 3 shows a schematic picture of the measurement.

As mentioned in the previous section, we truncate the sum over the eigenmodes in (4) at the $N$ th eigenvalue and denote the corresponding local condensate as $\bar{q} q^{(N)}(x)$. In the final analysis, we chose $N=160$, after confirming that the result is unchanged once a sufficient number of lowlying modes are included.

Reflecting the ultraviolet divergences of the scalar density operator, the expectation value of $\bar{q} q^{(N)}(x)$ contains quadratic and logarithmic divergences. The strong quadratic divergence is associated with a mixing with the identity operator and has the form $m_{q} / a^{2}$. Because of the exact chiral symmetry of the overlap-Dirac operator, the strongest divergence of $1 / a^{3}$ is absent, and the leading term is of $1 / a^{2}$ and proportional to $m_{q}$. Since the truncation at a fixed mode number $N$ can be considered as a certain regularization scheme, the regularized operator $\bar{q} q^{(N)}(x)$ can be parametrized as

$$
\bar{q} q^{(N)}=\bar{q} q^{(\text {subt })}+c_{1}^{(N)} m_{q} / a^{2}+c_{2}^{(N)} m_{q}^{3}
$$

with $\bar{q} q^{\text {(subt) }}$ the operator for which the power divergences are subtracted. The second and third terms represent a mixing with the identity operator; the mass dimension is compensated by $m_{q} / a^{2}$ and $m_{q}^{3}$, respectively. These coefficients $c_{1}^{(N)}$ and $c_{2}^{(N)}$ can be obtained by fitting the vacuum expectation value $\left\langle\bar{q} q^{(N)}\right\rangle$ as a function of the valence quark mass $m_{q}$ [26]. When the correlation with the Wilson loop is considered as in (10), the contribution from the identity operator with the divergent coefficient $c_{1}^{(N)} m_{q} / a^{2}+$ $c_{2}^{(N)} m_{q}^{3}$ cancels on the right-hand side, and the measurement is free from the power divergences.

Figure 4 shows the spatial distribution of $\left\langle\bar{q} q^{(N)}(\vec{x})\right\rangle_{W}$ on the $X Y$ plane with a separation $R=8$. The locations of color sources are shown by the circles. To improve the

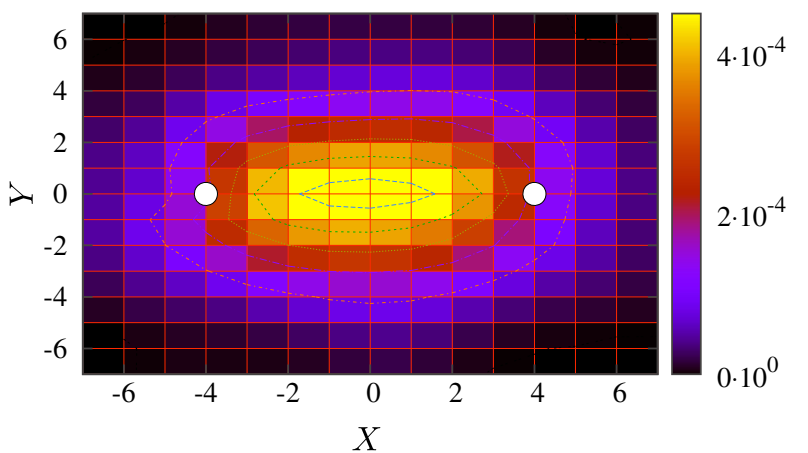

FIG. 4 (color online). Spatial profile of the local chiral condensate $\left\langle\bar{q} q^{(N)}(\vec{x})\right\rangle_{W}$ around a Wilson loop $W(R, T)$ with $R=8$ and $T=4$. The positions of color sources are at $(X, Y)=(4,0)$ and $(-4,0)$, which are shown in the plot by white circles.

signal of the Wilson loop, we apply the APE smearing for the spatial link variables [27], and the temporal extent is fixed at $T=4$ for which the ground state becomes dominant. In this plot, $\bar{q} q(\vec{x})$ is set at $t=0$, and the valence quark mass is $m_{q}=0.015 a^{-1}$.

To improve the signal, the lattice data are averaged over space-time. Namely, assuming the translational invariance of the expectation value, we shift the whole system including the Wilson loop and the local chiral condensate and take an average. This can be done without additional computational cost to solve quark propagators by using the low-lying eigenmodes. This is one of the advantages of the construction (4).

As Fig. 4 demonstrates, there appears a tubelike structure between the color sources, where the change of the condensate becomes positive, i.e., $\left\langle\bar{q} q^{(N)}(\vec{x})\right\rangle_{W}>0$. It means that the magnitude of the chiral condensate is reduced between the color charges, since $\langle\bar{q} q\rangle$ is negative in the vacuum.

Peak structures at the position of the charges are shown in the flux-tube measurements $[3,4]$ due to the strong enhancement of the action/energy density around the color charges. In terms of the low-mode truncated local chiral condensate shown in Fig. 4, no such characteristic structures around the color charges can be observed. The absence of peaks will be discussed later.

The remaining logarithmic divergence in $\bar{q} q^{(\text {subt })}$ can be canceled by taking a ratio

$$
r(\vec{x}) \equiv \frac{\left\langle\bar{q} q^{(\text {subt })}(\vec{x})\right\rangle_{W}}{\left\langle\bar{q} q^{(\text {subt })}\right\rangle}=\frac{\left\langle\bar{q} q^{(\text {subt })}(\vec{x}) W(R, T)\right\rangle}{\left\langle\bar{q} q^{(\text {subt })}\right\rangle\langle W(R, T)\rangle},
$$

where $\left\langle\bar{q} q^{\text {(subt) }}\right\rangle$ is obtained by fitting the vacuum expectation value $\langle\bar{q} q\rangle$ to (11) as a function of the valence quark mass $m_{q}$. As there are no remaining ultraviolet divergences, the ratio $r(\vec{x})$ has a proper continuum limit. Hereafter, we mainly use this quantity to quantitatively estimate the restoration of chiral symmetry. 
PARTIAL RESTORATION OF CHIRAL SYMMETRY IN THE ...

PHYSICAL REVIEW D 91, 094501 (2015)

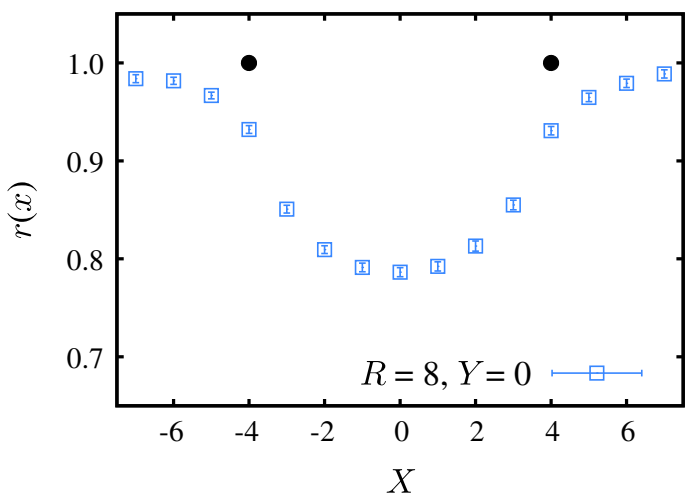

(a)

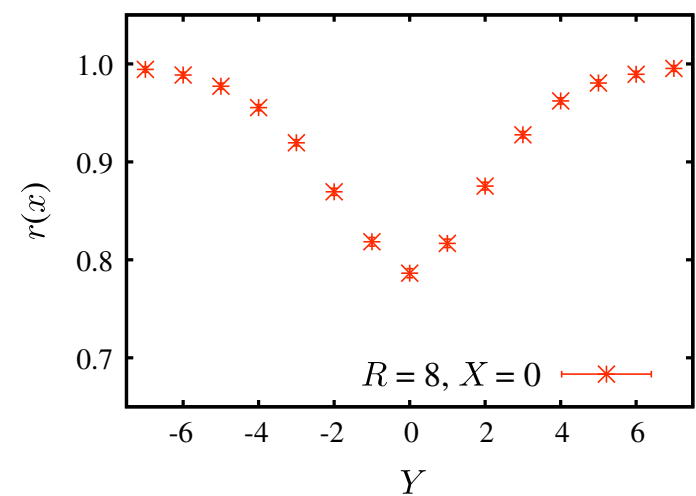

(b)

FIG. 5 (color online). Ratio of the chiral condensate $r(x)$ for a separation $R=8$. The color sources are separated along the $X$ axis and set at $(X, Y)=(4,0)$ and $(-4,0)$. The plots show the cross section (a) along the $X$ axis at $Y=0$ and (b) along $Y$ axis at $X=0$.

Figure 5 shows the ratio $r(\vec{x})$ for the separation between the color sources fixed at $R=8$. The plots Fig. 5(a) and Fig. 5(b) correspond to the cross sections of Fig. 4 along the $X$ axis and the transverse $Y$ axis. The locations of color sources are shown by black dots in Fig. 5(a).

These plots provide a quantitative measure of the reduction of the chiral condensate. The region in which the chiral condensate is reduced forms a structure that resembles the color flux tube. In other words, chiral symmetry is partially restored inside the flux tube. The restoration becomes stronger around the center of the flux, which is about $20 \%$ when $R=8$.

The close relationship between the flux tube is suggested in Fig. 6. We compare the cross section of both the chiral condensate $\left\langle\bar{q} q^{(\text {subt })}(\vec{x})\right\rangle_{W}$ and the action density defined by (8) with a cutoff on the mode number $\left\langle\rho^{(N)}(\vec{x})\right\rangle_{W}$ around the Wilson loop using 160 low-lying eigenmodes. The latter is calculated by inserting the action density $\rho(\vec{x})$ in place of $\bar{q} q(\vec{x})$ in Eq. (10), which is used for the flux-tube measurement [3-5]. To compare the profile, both quantities are normalized to unity at the origin. Apart from their normalization coefficients, the spatial profile of the chiral condensate shows a good agreement with UV Dirac mode truncated action density. As mentioned above, the action density is strongly enhanced around the color charges as reported in Refs. [3,4]. However, neither UV filtered density has such structures. Our conclusion is that such peak mainly comes from the ultraviolet divergent part and thus cannot be seen in Fig. 6(a) within our cutoff scale.

Figure 7 shows the same plot as Fig. 5 but with different values of $N$, the number of eigenmodes included in the sum (4). As expected from the construction that cancels the ultraviolet divergences, there is no significant difference between $N=120$ and 240 . Our choice $N=160$ is therefore sufficiently conservative to estimate the local chiral condensate inside the tube. Up to the largest eigenmode in our calculation at $N=240$, we have confirmed such a saturation for other quantities considered in this paper except for the magnitude of the action density $\rho^{(N)}(\vec{x})$ and topological charge density $q_{\mathrm{top}}^{(N)}(\vec{x})$. The value of these

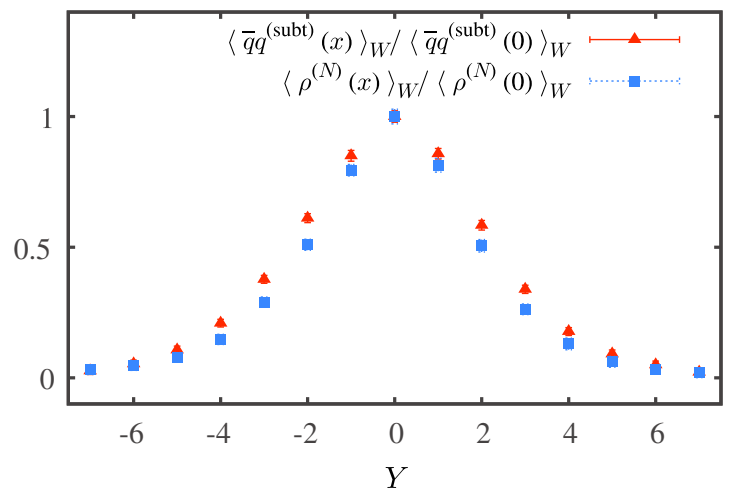

(b)

FIG. 6 (color online). The spatial profile of both the local chiral condensate $\left\langle\bar{q} q^{(\text {subt })}(\vec{x})\right\rangle_{W}$ and UV Dirac mode truncated action density $\left\langle\rho^{(N)}(\vec{x})\right\rangle_{W}$ around the color sources with a separation $R=8$ using 160 low-lying eigenmodes. For a comparison of their shape, both quantities are normalized at the origin. (a) cross section at $Y=0$. (b) cross section at $X=0$. 
TAKUMI IRITANI, GUIDO COSSU, AND SHOJI HASHIMOTO

PHYSICAL REVIEW D 91, 094501 (2015)

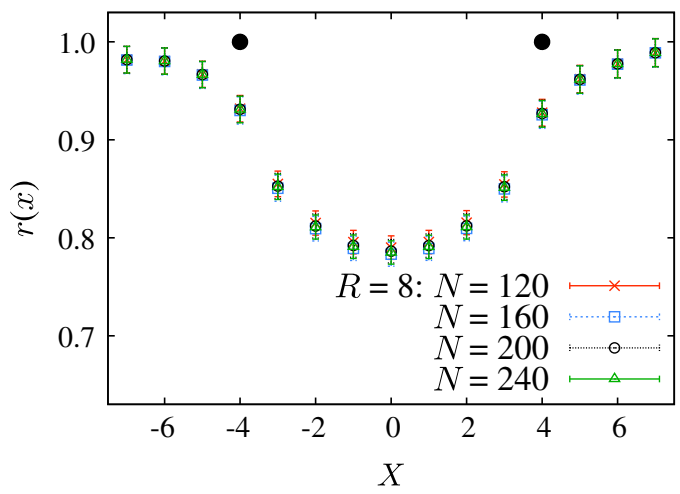

(a)

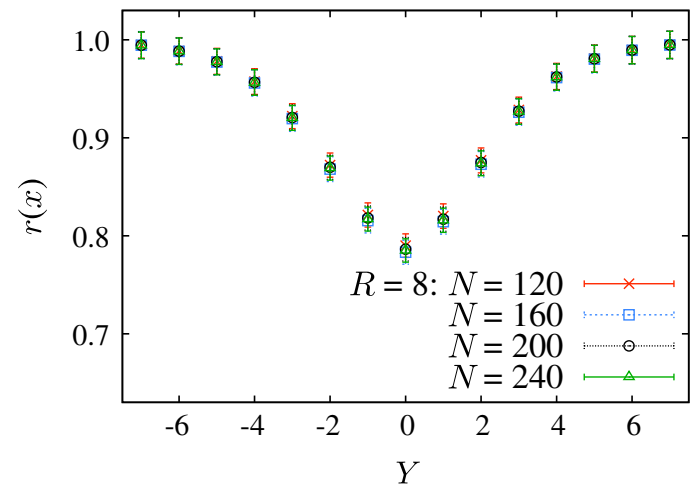

(b)

FIG. 7 (color online). Same as Fig. 5 but with different numbers of eigenmodes included: $N=120,160$, 200, and 240.(a) cross section at $Y=0$. (b) cross section at $X=0$.

quantities strongly depends on the cutoff scale $\lambda_{\max }$ as expected from the definition in Eqs. (8) and (9). However, the spatial profiles of both $\left\langle\rho^{(N)}(\vec{x})\right\rangle_{W}$ and $\left\langle\bar{q} q^{(\text {subt })}(\vec{x})\right\rangle_{W}$ are rather stable, and there are no signatures of peaks within our truncation as in Fig. 7(a).

The partial restoration of chiral symmetry is in accordance with the chiral bag model picture for the quarkantiquark system [28]. In the naive bag model, chiral symmetry is completely restored inside the bag, while Fig. 5 suggests a smooth boundary with a reduced but nonzero condensate inside the bag.

\section{B. Chiral symmetry restoration as a function of the separation}

Next, we study the chiral symmetry restoration depending on the separation of the color sources.

Figure 8 compares the cross section of $r(x)$ along the $X$ axis with $R=4,8$, and 10 . By increasing the separation, we observe that the region of partial restoration stretches between the color sources, which are located at $X=R / 2$ and $-R / 2$. This supports the picture of the tube structure.

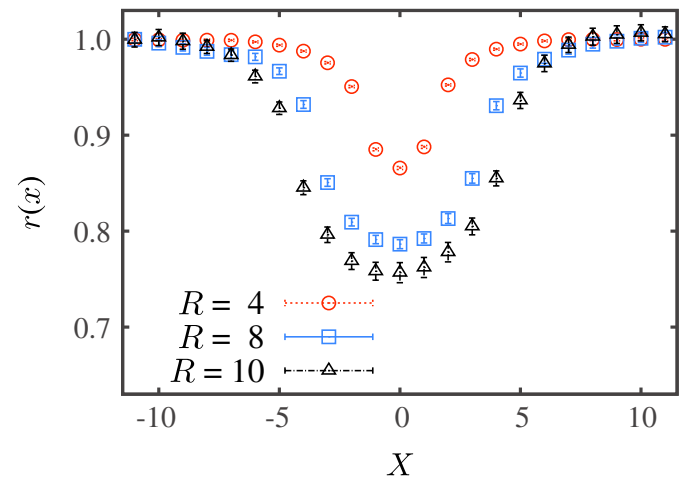

FIG. 8 (color online). Chiral condensate ratio $r(x)$ along the $X$ axis. The results with increasing separation $R: R=4,8$, and 10. Color sources are located at $(R / 2,0)$ and $(-R / 2,0)$.
The magnitude of the reduction increases with $R$. For instance, at the origin, the reduction of about $15 \%$ at $R=4$ grows up to about $25 \%$ at $R=10$. Beyond $R=10$, the statistical signal becomes much worse, and the effect of spatial boundary would become important as $R$ approaches $L / 2$.

In Fig. 9, we plot the value of the ratio at the center $r(0)$, where the magnitude becomes minimum, as a function of $R$. As the separation $R$ increases, the ratio of the chiral condensate decreases monotonically until the maximum distance we could explore. At larger distances, the effect of string breaking should manifest itself in dynamical QCD, and the local chiral condensate would stop decreasing. As far as we can observe, the reduction of the chiral condensate inside the color flux tube is of the size of $20 \%-25 \%$ at the distance of $1 \mathrm{fm}$, assuming that the string breaking does not occur in this scale [29], since it is difficult to observe the breaking state using the Wilson loop as a color source.

By increasing the separation between color sources, the thickness of the flux is expected to grow logarithmically as a function of its length [30,31]. Such behavior has indeed been observed in quenched lattice QCD calculations [32]

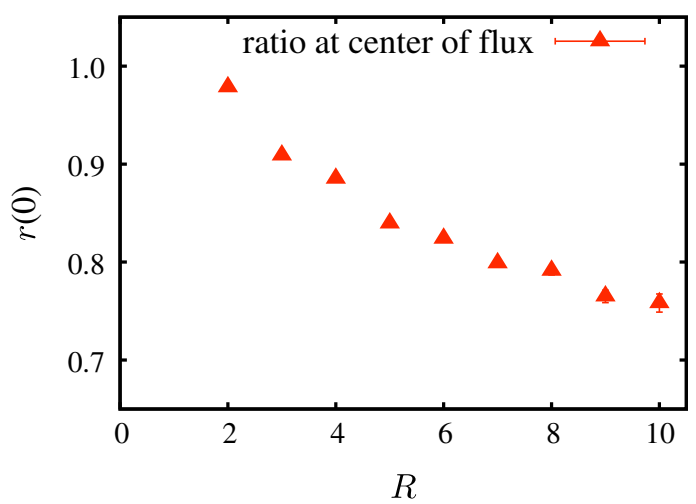

FIG. 9 (color online). Ratio at the center of the flux $r(0)$ as a function of the separation $R$. 


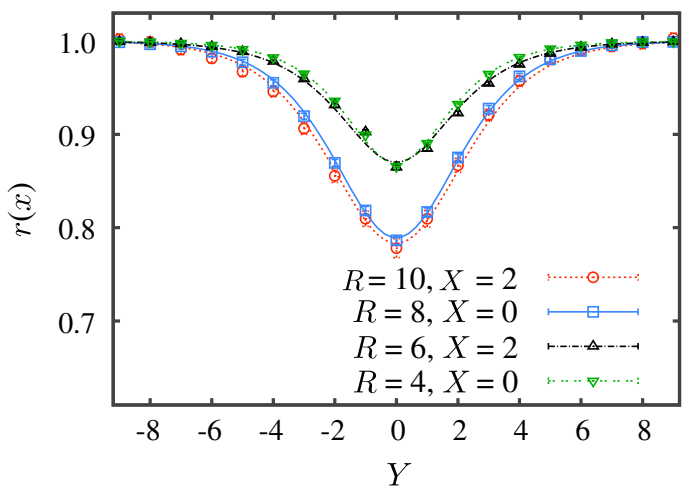

FIG. 10 (color online). Cross section of the ratio along the $Y$ axis with different separations $R$ and cut points $X$.

(see also Ref. [33] for a study at finite temperature), for which one can increase the statistics more easily. In this work, we try to observe the thickness through the chiral condensate ratio $r(x)$.

Figure 10 shows the cross section of $r(x)$ along the $Y$ axis for some combinations of $R$ and $X$. It is clear that for a fixed $X$ the flux is thicker when the separation $R$ is larger. More interestingly, the curve for $R=8$ at $X=0$ almost coincides with that for $R=10$ at $X=2$. Similarly, the curve for $R=4$ at $X=0$ coincides with that for $R=6$ at $X=2$. Because of the reflection symmetry, these behaviors are also observed at $X=-2$. This indicates that the thickness of the flux is highly correlated with the magnitude of the reduction. We also note that these corresponding cross sections have the same distance from the color charge, which is given by $R / 2-|X|$ for $|X| \leq R / 2$.

In fact, such a coincidence is expected from an effective string model $[34,35]$. According to that model, the ratio $r(Y)$ is written as

$$
r(Y)=1-\tilde{r} \frac{\mu^{2}}{\alpha} \frac{K_{0}\left(\left(\mu^{2} Y^{2}+\alpha^{2}\right)^{1 / 2}\right)}{K_{1}(\alpha)},
$$

TABLE I. Fit results for $r(Y)$ to (13) for each $R$ and $X$, together with $\chi^{2}$ per degrees of freedom. Note that $\mu$ and $\alpha$ are in lattice units. The condensate ratio at the center $r(0)$ is listed as well.

\begin{tabular}{lcccccc}
\hline \hline$R$ & $X$ & \multicolumn{1}{c}{$r(0)$} & $\tilde{r}$ & \multicolumn{1}{c}{$\mu$} & \multicolumn{1}{c}{$\alpha$} & $\chi^{2} /$ dof \\
\hline 10 & 0 & $0.757(10)$ & $1.54(7)$ & $0.66(11)$ & $2.3(0.9)$ & 0.40 \\
10 & 1 & $0.762(10)$ & $1.46(7)$ & $0.72(14)$ & $2.8(1.2)$ & 0.46 \\
10 & 2 & $0.778(10)$ & $1.26(6)$ & $0.71(14)$ & $2.5(1.1)$ & 0.66 \\
10 & 3 & $0.805(9)$ & $1.00(6)$ & $0.75(18)$ & $2.5(1.3)$ & 1.00 \\
8 & 0 & $0.786(5)$ & $1.11(3)$ & $0.71(7)$ & $2.2(0.5)$ & 0.43 \\
8 & 1 & $0.792(5)$ & $1.08(4)$ & $0.72(8)$ & $2.3(0.6)$ & 0.25 \\
8 & 2 & $0.813(5)$ & $0.93(3)$ & $0.75(11)$ & $2.5(0.8)$ & 0.58 \\
8 & 3 & $0.855(5)$ & $0.69(3)$ & $0.83(17)$ & $3.0(1.3)$ & 1.42 \\
6 & 0 & $0.815(3)$ & $0.89(2)$ & $0.66(4)$ & $1.7(2)$ & 0.38 \\
6 & 1 & $0.827(3)$ & $0.81(2)$ & $0.65(4)$ & $1.6(2)$ & 1.01 \\
6 & 2 & $0.865(3)$ & $0.65(2)$ & $0.61(4)$ & $1.4(2)$ & 2.36 \\
\hline \hline
\end{tabular}

where $K_{0}(x)$ and $K_{1}(x)$ are modified Bessel functions. The parameter $\mu$ has a physical interpretation as the inverse penetration length of the flux from the perpendicular direction, and $\alpha$ is the thickness of the core. The parameter $\tilde{r}$ represents the strength of the condensate reduction.

The function (13) reproduces the lattice data quite well, as shown by the curves in Fig. 10. The fit results are summarized in Table I. The penetration length is in the range of $1 / \mu \simeq 1.0-1.6$, which corresponds to $0.11-0.18 \mathrm{fm}$ in physical units. The core size $\alpha$ is $1.4-3.6$ in lattice units and is in the range $0.15-0.4 \mathrm{fm}$. We observe an increase of $\alpha$ as $R$ increases while $X$ is fixed at zero, but with the large statistical error, we are not able to claim the clear evidence of the string fattening.

\section{CHIRAL CONDENSATE IN THE THREE-QUARK SYSTEM}

\section{A. Partial restoration of chiral symmetry in the three-quark system}

Next, we consider a system consisting of three color charges that represents a baryon system, which we call the 3Q system in this paper.

Using the path-ordered product $U_{k} \equiv \prod_{\Gamma_{k}} e^{i a g A_{k}}$ along a path $\Gamma_{k}$, the 3Q Wilson loop is given by

$$
W_{3 \mathrm{Q}} \equiv \frac{1}{3} \varepsilon_{a b c} \varepsilon_{a^{\prime} b^{\prime} c^{\prime}} U_{1}^{a a^{\prime}} U_{2}^{b b^{\prime}} U_{3}^{c c^{\prime}}
$$

which is made color singlet by the totally antisymmetric tensor $\varepsilon_{a b c}$ of color indices $a, b$, and $c$ [36,37]. Similar to the QQ system, the spatial distribution of the chiral condensate for the $3 \mathrm{Q}$ system is measured as

$$
\langle\bar{q} q(\vec{x})\rangle_{3 \mathrm{Q}} \equiv \frac{\left\langle\bar{q} q(\vec{x}) W_{3 \mathrm{Q}}\right\rangle}{\left\langle W_{3 \mathrm{Q}}\right\rangle}-\langle\bar{q} q\rangle,
$$

with the $3 \mathrm{Q}$ Wilson loop $W_{3 \mathrm{Q}}$. The ratio of the chiral condensate in the 3Q system $r_{3 \mathrm{Q}}(\vec{x})$, for which the ultraviolet divergences cancel, is then constructed by

$$
r_{3 \mathrm{Q}}(\vec{x}) \equiv \frac{\left\langle\bar{q} q^{(\text {subt })}(\vec{x}) W_{3 \mathrm{Q}}\right\rangle}{\left\langle\bar{q} q^{(\text {subt })}\right\rangle\left\langle W_{3 \mathrm{Q}}\right\rangle} .
$$

Figure 11 shows a schematic picture of the construction of the 3Q Wilson loop $W_{3 \mathrm{Q}}$ from the Wilson lines $U_{k}$. For simplicity, we use an isosceles right triangle configuration of the color charges on the $X Y$ plane, and the coordinate is set as in Fig. 11. In this case, the junction point of the three flux tubes (the Fermat point) corresponds to the origin $[36,37]$. The measurement of the local chiral condensate $\bar{q} q(\vec{x})$ is done at a fixed time slice. The low-mode truncation number $N$, the temporal extension $T$, and other measurement setups are the same as in the QQ system. 
3Q Wilson Loop

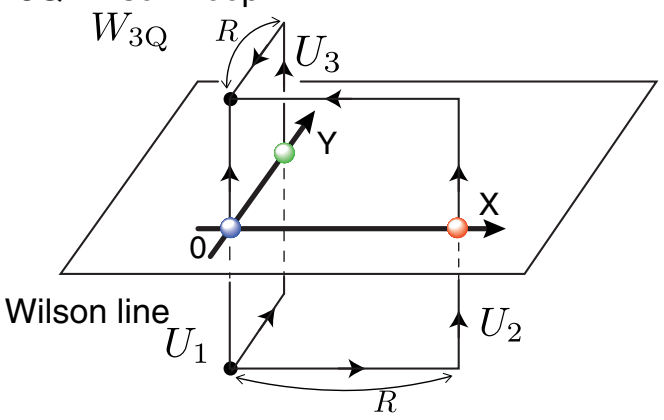

FIG. 11 (color online). A schematic picture of the construction for three-quark system with an isosceles right triangle configuration. Three Wilson lines $U_{k}$ correspond to the static color sources.

Figure 12 shows the ratio $r_{3 \mathrm{Q}}(\vec{x})$ with color sources at $(X, Y)=(6,0),(0,6)$, and $(0,0)$ denoted by circles in the plot. As shown in Fig. 12, the magnitude of the chiral condensate is reduced among the color sources, which indicates the partial restoration of chiral symmetry inside the 3Q system. Similar to the QQ system in Sec. III, there appear no peaks at the color charges within our truncation scale. We note that the characteristic $Y$-type flux is not clearly seen in this plot, probably because the thickness of the flux is comparable to the color source separation. Because of the statistical noise, we are not able to repeat the calculations increasing the quark separations.

Like in the QQ system, the magnitude of the restoration depends on the separation of the sources. Figure 13 shows the cross section of the ratio $r_{3 \mathrm{Q}}(\vec{x})$ along the line of $X=Y$ with the color sources at $(X, Y)=(R, 0),(0, R)$, and $(0,0)$. In this setup, the measurement goes through one color charge and the center of mass of the system. By comparing the data for $R=3$ and 6 , we find that the reduction is more substantial for $R=6$, which is similar to the QQ $\bar{Q}$ system (see Fig. 8). The reduction of the local chiral condensate becomes larger with the size of the loop and takes its minimum value at around the center of gravity. With $R=6$,

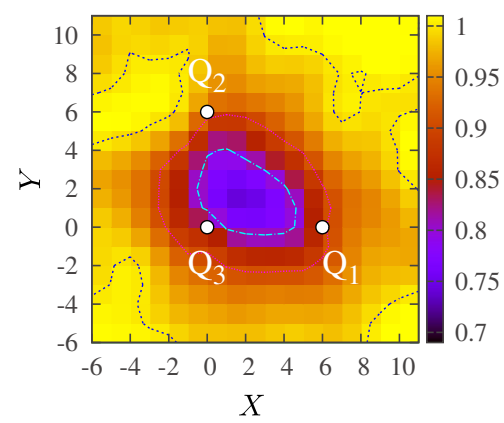

FIG. 12 (color online). Condensate ratio $r_{3 \mathrm{Q}}(\vec{x})$ with the color sources at $Q_{1}=(6,0), Q_{2}=(0,6)$, and $Q_{3}=(0,0)$ on the $X Y$ plane.

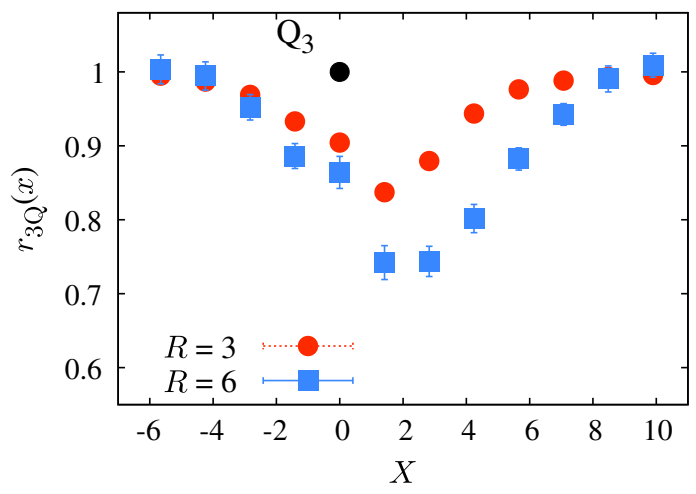

FIG. 13 (color online). Chiral condensate ratio $r_{3 \mathrm{Q}}(\vec{x})$ along the line of $X=Y$ with the color sources at $(R, 0),(0, R)$, and $(0,0)$ on the $X Y$ plane with $R=3$ and 6 .

the reduction is about $30 \%$, which is also similar to that of the QQ system.

\section{B. Partial restoration at finite density}

Finally, using the observed modification of the local chiral condensate around the color sources, we estimate the size of the partial restoration of chiral symmetry in finitedensity QCD. We consider the system of a fixed number of baryons in a finite-volume box so that the baryon number density $\rho$ is $N_{b} / L^{3}$, where $N_{b}$ is the number of baryons and $L^{3}$ is the spatial volume. As a toy example, we take $N_{b}=1$ and replace the baryon by the $3 \mathrm{Q}$ Wilson loop. This only gives a crude approximation of the realistic system, but given the difficulty of simulating QCD at finite chemical potential, it may provide a useful clue to the understanding of the finite-density QCD.

The net change of the condensate under such a system is estimated by the spatial average of the condensate ratio $r_{3 \mathrm{Q}}(\vec{x})$,

$$
\frac{\langle\bar{q} q\rangle_{\rho}}{\langle\bar{q} q\rangle_{0}} \equiv \frac{1}{L^{3}} \sum_{\vec{x}}^{L^{3}} r_{3 \mathrm{Q}}(\vec{x}),
$$

where $\langle\bar{q} q\rangle_{\rho}$ is the condensate at the finite baryon number density $\rho=1 / L^{3}$. We use two lattice volumes, $L^{3}=16^{3}$ and $24^{3}$, which correspond to $(16 a)^{-3} \simeq 0.18 \mathrm{fm}^{-3}$ and $(24 a)^{-3} \simeq 0.05 \mathrm{fm}^{-3}$, respectively. The $16^{3}$ lattice roughly corresponds to the normal nuclear density $\rho_{0} \simeq 0.18 \mathrm{fm}^{-3}$.

Figure 14 shows $\langle\bar{q} q\rangle_{\rho} /\langle\bar{q} q\rangle_{0}$ as a function of $1 / L^{3}$. The two symbols correspond to the different configurations of the color sources, i.e., $(0,0),(R, 0)$, and $(0, R)$ with $R=3$ and 6 on the $X Y$ plane. The solid lines are the results of a linear fit with fixed value of 1 at $1 / L^{3}=0$. The linear dependence from unity at $\rho=0$ simply means that there is a finite region where the chiral condensate is reduced from its vacuum value. Since the region gets larger with increasing $R$, the slope for larger $R$ is steeper.

In our setup, the reduction of the chiral condensate at the normal nuclear density is only $\sim 5 \%$, which is much smaller 


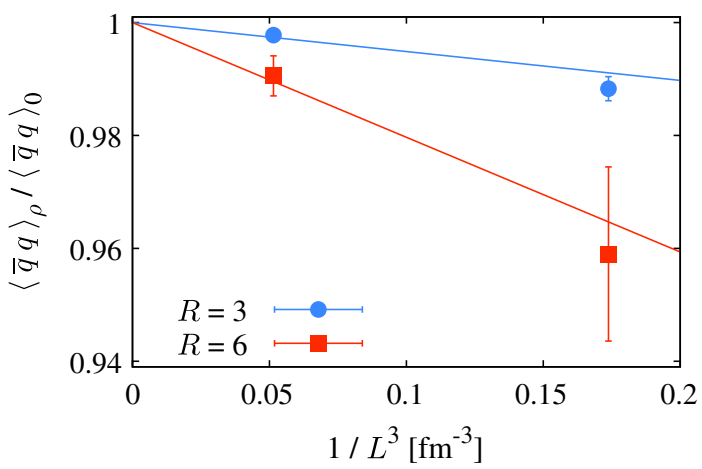

FIG. 14 (color online). Reduction of the chiral condensate at finite density measured by $\langle\bar{q} q\rangle_{\rho} /\langle\bar{q} q\rangle_{0}$. The estimates for a given configuration of color sources, i.e., at $(0,0),(R, 0)$, and $(0, R)$ on the $X Y$ plane, with $R=3$ and 6 are shown.

than the phenomenological model estimate of the order of $30 \%$ [38]. Our estimate, however, assumes a fixed spatial size of the baryon that is smaller than the realistic nucleon. For instance, the mean root square radius of our setup in Fig. 14 is $0.44 \mathrm{fm}$ when $R=6$, while the charge radius of proton is $0.88 \mathrm{fm}$. As the restoration of the chiral condensate is stronger for larger separation, this suggests that $\langle\bar{q} q\rangle_{\rho}$ in realistic finite-density QCD could be substantially lower than our estimate.

\section{SUMMARY}

The Dirac eigenmodes carry the full information of the background gauge field. Indeed, having the complete set of the eigenvalue and eigenvectors, one can reconstruct the field-strength tensor $F_{\mu \nu}(x)$ at any point $x$. They therefore offer an interesting way of filtering out the ultraviolet modes and investigating the low-energy dynamics of QCD by only using the low-lying eigenmodes upon reconstruction. This is a sound and well-defined regularization method of quantum field theory.

We use this method to investigate the spatial profile of the chiral condensate under the presence of external sources. On the lattices generated with $2+1$ flavors of dynamical overlap fermions, we calculate the low-lying eigenvalues and associated eigenvectors of the overlapDirac operator and use them to reconstruct the chiral condensate locally. Then, it is straightforward to measure its correlation with the external color sources set up to model the QQ and 3Q systems.

We find that the local chiral condensate shows a structure interpreted as a color flux tube between the QQQ color sources, in which the condensate decreases significantly. It indicates a partial restoration of chiral symmetry inside the flux tube and suggests that it happens also inside hadrons. The spatial profile is consistent with a string model of the confinement potential, giving another support for the presence of the color flux tube.

We perform a similar measurement in the $3 \mathrm{Q}$ system, which is new as far as we have noticed. It again shows the partial restoration of chiral symmetry among the color sources. The reduction of the condensate is about $30 \%$ for the separation between the color sources of $\sim 1 \mathrm{fm}$. It can be used to estimate the chiral condensate in the finite-density system.

The method developed in this work may easily be applied for the study of finite-temperature QCD, in which Polyakov loops can be used for a static color source. Since the eigenmodes can be applied to define various charges, such as the axial charge density, the quark number density, and the topological charge, it may provide an interesting alternative to measure their spatial distribution.

\section{ACKNOWLEDGMENTS}

The lattice QCD calculations have been done on SR16000 at High Energy Accelerator Research Organization (KEK) under a support of its Large Scale Simulation Program (Program No. 13/14-05). This work is supported in part by JSPS KAKENHI Grants No's. 25287046 and 26247043, and the Strategic Program for Innovative Research (SPIRE) Field 5 project.
[1] T. Banks and A. Casher, Nucl. Phys. B169, 103 (1980).

[2] K. Fukushima and T. Hatsuda, Rep. Prog. Phys. 74, 014001 (2011).

[3] G. S. Bali, K. Schilling, and C. Schlichter, Phys. Rev. D 51, 5165 (1995).

[4] R. W. Haymaker, V. Singh, Y. C. Peng, and J. Wosiek, Phys. Rev. D 53, 389 (1996).

[5] P. Cea and L. Cosmai, Phys. Rev. D 52, 5152 (1995).

[6] C. Gattringer, Phys. Rev. Lett. 88, 221601 (2002).
[7] S. Aoki, T.-W. Chiu, G. Cossu, X. Feng, H. Fukaya, S. Hashimoto, T.-H. Hsieh, T. Kaneko, H. Matsufuru, J.-I. Noaki, T. Onogi, E. Shintani, and K. Takeda, Prog. Theor. Exp. Phys. 2012, 01A106 (2012).

[8] H. Neuberger, Phys. Lett. B 417, 141 (1998).

[9] H. Neuberger, Phys. Lett. B 427, 353 (1998).

[10] H. Fukaya et al. (JLQCD Collaboration), Phys. Rev. Lett. 98, 172001 (2007).

[11] H. Fukaya, S. Aoki, T. W. Chiu, S. Hashimoto, T. Kaneko, H. Matsufuru, J. Noaki, K. Ogawa, T. Onogi, and N. 
Yamada (JLQCD and TWQCD Collaborations), Phys. Rev. D 76, 054503 (2007).

[12] H. Fukaya, S. Aoki, S. Hashimoto, T. Kaneko, J. Noaki, T. Onogi, and N. Yamada (JLQCD Collaboration), Phys. Rev. Lett. 104, 122002 (2010); 105, 159901(E) (2010).

[13] H. Fukaya, S. Aoki, T. W. Chiu, S. Hashimoto, T. Kaneko, J. Noaki, T. Onogi, and N. Yamada (JLQCD and TWQCD Collaborations), Phys. Rev. D 83, 074501 (2011).

[14] T. Iritani, G. Cossu, and S. Hashimoto, Proc. Sci., LATTICE2013 (2014) 376.

[15] T. Iritani, G. Cossu, and S. Hashimoto, Proc. Sci., Hadron2013 (2013) 159.

[16] T. Iritani, G. Cossu, and S. Hashimoto, Proc. Sci., Lattice2014 (2014) 338.

[17] F. Niedermayer, Nucl. Phys. B, Proc. Suppl. 73, 105 (1999).

[18] H. Fukaya, S. Hashimoto, K.-I. Ishikawa, T. Kaneko, H. Matsufuru, T. Onogi, and N. Yamada (JLQCD Collaboration), Phys. Rev. D 74, 094505 (2006).

[19] S. Aoki, H. Fukaya, S. Hashimoto, and T. Onogi, Phys. Rev. D 76, 054508 (2007).

[20] E.-M. Ilgenfritz, K. Koller, Y. Koma, G. Schierholz, T. Streuer, and V. Weinberg, Phys. Rev. D 76, 034506 (2007).

[21] E.-M. Ilgenfritz, D. Leinweber, P. Moran, K. Koller, G. Schierholz, and V. Weinberg, Phys. Rev. D 77, 074502 (2008); 77, 099902(E) (2008).

[22] W. Feilmair, M. Faber, and H. Markum, Phys. Rev. D 39, 1409 (1989).

[23] W. Sakuler, W. Burger, M. Faber, H. Markum, M. Müller, P. De Forcrand, A. Nakamura, and I. O. Stamatescu, Phys. Lett. B 276, 155 (1992).
[24] M. Faber, M. Schaler, and H. Gausterer, Phys. Lett. B 317, 409 (1993).

[25] S. Chagdaa and E. Laermann, Proc. Sci., LAT 2007 (2007) 172.

[26] J. Noaki, T. W. Chiu, H. Fukaya, S. Hashimoto, H. Matsufuru, T. Onogi, E. Shintani, and N. Yamada, Phys. Rev. D 81, 034502 (2010).

[27] M. Albanese et al. (APE Collaboration), Phys. Lett. B 192 , 163 (1987).

[28] A. Hosaka and H. Toki, Phys. Rep. 277, 65 (1996).

[29] G. S. Bali, H. Neff, T. Düssel, T. Lippert, and K. Schilling (SESAM Collaboration), Phys. Rev. D 71, 114513 (2005).

[30] A. Hasenfratz, E. Hasenfratz, and P. Hasenfratz, Nucl. Phys. B180, 353 (1981).

[31] M. Lüscher, G. Münster, and P. Weisz, Nucl. Phys. B180, 1 (1981).

[32] N. Cardoso, M. Cardoso, and P. Bicudo, Phys. Rev. D 88, 054504 (2013).

[33] A. S. Bakry, D. B. Leinweber, and A. G. Williams, Phys. Rev. D 85, 034504 (2012).

[34] P. Cea, L. Cosmai, and A. Papa, Phys. Rev. D 86, 054501 (2012).

[35] D. E. Kharzeev and F. Loshaj, Phys. Rev. D 90, 037501 (2014).

[36] T. T. Takahashi, H. Matsufuru, Y. Nemoto, and H. Suganuma, Phys. Rev. Lett. 86, 18 (2001).

[37] T. T. Takahashi, H. Suganuma, Y. Nemoto, and H. Matsufuru, Phys. Rev. D 65, 114509 (2002).

[38] R. S. Hayano and T. Hatsuda, Rev. Mod. Phys. 82, 2949 (2010). 